\title{
Autoconcepto y tendencia religiosa en universitarios: propiedades psicométricas del $\mathrm{AF}-5^{*}$
}

\section{Self-Concept and Religious Tendency in University Students: Psychometric Properties of AF-5}

\author{
FÉLIX Zurita-Ortega \\ Universidad de Granada, España \\ ORCID: http://orcid.org/0000-0002-1189-894X \\ Silvia San Román-Mata \\ Universidad de Granada, España \\ ORCID: http://orcid.org/0000-0003-1633-2926 \\ Asunción Martínez-Martínez \\ Universidad de Granada, España \\ ORCID: http://orcid.org/0000-0002-8826-235X \\ Ramón Chacón-Cuberos ${ }^{\mathrm{a}}$ \\ Universidad de Granada, España \\ ORCID: http://orcid.org/0000-0003-0937-1089 \\ Manuel Castro-Sánchez \\ Universidad de Granada, España \\ ORCID: http://orcid.org/0000-0002-2357-3093 \\ Pilar Puertas-Molero \\ Universidad de Granada, España \\ ORCID: http://orcid.org/0000-0001-8878-7677
}

a Autor de correspondencia. Correo electrónico: rchacon@ugr.es

Para citar este artículo: Zurita-Ortega, F., San RománMata, S., Martínez-Martínez, A., Chacón-Cuberos, R., Castro-Sánchez, M., \& Puertas-Molero, P. (2018). Autoconcepto y tendencia religiosa en universitarios: propiedades psicométricas del AF-5. Autoconcepto Forma 5. Universitas Psychologica, 17(5), 1-12. http:// doi.org/10.11144/Javeriana.upsy17-5.atru

\section{RESUMEN}

El objetivo de esta investigación ha sido analizar las propiedades psicométricas del cuestionario AF-5 mediante técnicas de tipo exploratorio y confirmatorio en universitarios de diferentes tendencias religiosas, así como analizar la relación entre el autoconcepto y tendencia religiosa (cristianos, musulmanes y ateo-agnósticos). La muestra estuvo formada por 597 estudiantes (23.04 \pm 3.72 años), donde 156 (26.1 $\%)$ eran hombres y 441 (73.9 \%) mujeres, empleando como principales instrumentos el test de Autoconcepto AF-5 y una hoja de autorregistro para la tendencia religiosa. Los resultados indican que el cuestionario ofrece índices de fiabilidad superiores a $\alpha=0.7$. La factorialidad se ajusta adecuadamente al modelo de cinco factores original. Asimismo, las dimensiones académica y emocional presentan una mayor fortaleza en los cristianos, mientras que la social lo hace en los musulmanes. Las dimensiones familiar y física son más elevadas para universitarios que profesan alguna religión.

Palabras clave

autoconcepto; religión; estudiantes universitarios. 


\begin{abstract}
This research aims to analyse the psychometric properties of AF-5 questionnaire using exploratory and confirmatory techniques in university students of different religious tendencies, as well as to examine the relationship between self-concept and religious tendency (Christian, Muslim and atheist-agnostics). The sample consisted of 597 students (23.04 \pm 3.72 years old), where 156 (26.1\%) were men and 441 (73.9\%) were women, using as main instruments the AF-5 questionnaire and a self-registration sheet for religious tendendy. Results showed that AF-\% questionnaire offers reliability indexes greater than $\alpha=$ 0.7. In addition, factors are properly adjusted with the original model with five factors. Furthermore, the academic and emotional dimensions of self-concept displayed a greater strength in Christians, whereas social dimension was stronger in Muslims. Family and physical dimensions were higher for university students who profess some religion.

Keywords

self-concept; religion; university students.
\end{abstract}

La percepción que un individuo posee de sí mismo (autoconcepto) resulta de interés a nivel individual (físico, social o espiritual) y colectivo (social). En los últimos años a nivel mundial, se ha experimentado un importante auge sobre esta temática en la literatura, con el fin de dilucidar aspectos que permitan desarrollar y extrapolar este constructo a otras áreas (García \& Musitu, 1999; Wilkins, Butt, Kratochvil, \& Balakrishnan, 2016). De hecho, casi todos los autores coinciden en que las actitudes y comportamientos que se adquieren en la etapa adolescente y postadolescente repercuten en la edad adulta, y el autoconcepto opera en este proceso constructivo.

En ese sentido, este es un tema abordado por multitud de estudios en la actualidad. Algunos de ellos lo analizan desde los aspectos sociales (Fernández-Zabala, RodríguezFernández, \& Goñi, 2016) o emocionales (Tornare, Czajkowski, \& Pons, 2015). Otros, desde el análisis académico (Lohbeck, Nitkowski, \& Petermann, 2016), psicológico (Flores, Medrano, \& Manoiloff, 2014) o físico-deportivo (Zurita, Castro-Sánchez, Álvaro, Rodríguez, \& Pérez, 2016). El fenómeno religioso, en sus diversas manifestaciones, se encuentra presente en numerosas disciplinas del conocimiento (filosofía, sociología, educación o psicología) y puede configurar ámbitos de investigación específicos (Worthen, Lingiardi, \& Caristo, 2017). Se ha constatado un creciente interés en los últimos años por su estudio (Exline, Pargament, Grubbs, \& Yali, 2014; Grubbs, Wilt, Stauner, Exline, \& Pargament, 2016), abordándose desde las expresiones sociales (López, Huynh, \& Fuligni, 2011), personales o del bienestar (Pargament, 2002), entre otras. $\mathrm{Si}$ bien los indicadores sociológicos muestran que la religión es un fenómeno a la baja desde hace más de una década en el continente europeo y particularmente en España (Elzo \& González-Anleo, 1999), estos indican que no se debe obviar que la tendencia religiosa es una dimensión estrictamente aplicable al espacio personal y emocional del individuo, sin necesidad de presentar un vínculo con la moral o con el aspecto político. Varios autores señalan que el ser humano desarrolla una concepción del mundo que se relaciona con el perfil estimativo o axiológico, acciones morales o tipo de religiosidad (Grubbs et al., 2016; Pérez-Delgado, 2000; Puertas-Molero, GonzálezValero, \& Sánchez-Zafra, 2017), e interfiere en la noción de la persona sobre sí misma. Desde la perspectiva del bienestar personal que produce este término, el autoconcepto se puede referir a las etiquetas que una persona se atribuye, generalmente relacionado con parámetros físicos, comportamentales y emocionales. Desde que Wylie (1979) criticara la concepción unidimensional del modelo de tipo jerárquico planteado por Shavelson, Hubner y Stanton (1976), la gran mayoría de autores consideran este constructo de forma multidimensional, manifestando la importancia y el bienestar que ocasiona hacia la persona, como señalan Marsh (1990), Standage y Treasure (2002), Standage, Duda y Ntoumanis (2005) o Zurita et al. (2018).

En la última década se ha generado una adaptación del test de Autoconcepto Forma 5 (AF5) a diversos colectivos (García, Gracia, \& Zeleznova, 2013), pero son escasos los que han empleado la adaptación de este instrumento en universitarios (Lobos, Díaz, Bustos, \& Pérez, 2015; Silvia, 2016). Asimismo, resulta 
importante mencionar que algunos autores eliminan o suprimen factores en las validaciones del AF-5 (Núñez, Martín-Albo, Navarro, \& Grijalvo, 2007; Silvia, 2016).

Se observa una escasez de trabajos que aborden las asociaciones entre la tendencia religiosa y el autoconcepto, destacando únicamente el estudio realizado por Grubbs et al. (2016). En este sentido, se encuentra en esta área un campo fértil para la investigación acerca del cual discurre el presente documento, y especialmente si se preconcibe que la actitud religiosa podría intervenir de una forma directa sobre las dimensiones del autoconcepto.

Por tanto, parece que el modelo de cinco factores no ha sido probado suficientemente mediante técnicas de tipo exploratorio y confirmatorio, en personas de diferente concepción y actitud religiosa. El objetivo del estudio ha sido analizar y describir las propiedades psicométricas del AF5, para comprobar la fiabilidad del instrumento con la finalidad de su adaptación y aplicación a la población de estudiantes universitarios de diversa tendencia religiosa, así como estudiar la relación existente entre el autoconcepto y la tendencia religiosa.

\section{Método}

\section{Diseño y participantes}

En este estudio, participaron 597 estudiantes universitarios de grado de entre 19 y 23 años (23.04 \pm 3.72 años de edad), donde 156 (26.1 \%) eran hombres y 441 (73.9 \%) mujeres. Los participantes procedían de tres ciudades donde la Universidad de Granada (España) tiene campus universitarios, distribuyéndose de la siguiente forma: 103 en Granada (17.3\%), 138 de Ceuta (23.1 \%) y 356 de Melilla (59.6\%). La muestra se obtuvo mediante un muestreo aleatorio simple. Se debe indicar que se detectaron 28 cuestionarios erróneos en su realización que fueron excluidos del estudio.

\section{Variables e instrumentos}

Para el desarrollo de este trabajo de investigación, se utilizaron dos instrumentos. El primero de ellos fue un cuestionario de tipo sociodemográfico en el cual se determina el sexo (hombre o mujer), edad, campus y titulación, e inclinación religiosa (cristiana, musulmana o ateo-agnóstica), según lo planteado por Worthen et al. (2017). El segundo cuestionario administrado fue el Autoconcepto Forma-5 (AF-5) de García y Musitu (1999). Este instrumento se fundamenta en el modelo teórico de Shavelson et al. (1976) y está conformado por 30 preguntas agrupadas en cinco dimensiones: académico (ítems $1,6,11$, 16,21 y 26), social (ítems $2,7,12,17,22$ y 27), emocional (ítems 3, 8, 13, 18, 23 y 28), familiar (ítems 4, 9, 14, 19, 24 y 29) y físico (ítems 5, 10, $15,20,25$ y 30$)$. Los participantes respondieron en una escala entre 1 y 5 , donde el $1=$ nunca y $5=$ siempre. Esta forma de respuesta ha sido adaptada, entre otros, por Salum-Fares, Marín y Reyes (2011) y Zurita et al. (2016), aunque en su origen la medición de los ítems oscilaba entre 1 y 99.

\section{Procedimiento}

Los cuestionarios fueron administrados por los investigadores preparados para tal efecto, en horario no lectivo, de manera grupal, después de obtener el consentimiento informado de los propios estudiantes. Se garantizó el anonimato de la información recogida a los participantes, aclarando que su utilización sería solo con fines científicos. Con el fin de evitar respuestas no sinceras y reducir lo máximo posible el efecto de deseabilidad social, los participantes desconocían la finalidad del estudio.

\section{Análisis de datos}

En este estudio, las propiedades psicométricas (fiabilidad y análisis factorial exploratorio) y análisis descriptivos básicos se realizaron mediante el paquete estadístico SPSS 22.0 para 
Windows y el Programa FACTOR Analysis 9.3.1 (González \& Ibáñez, 2018; Lorenzo-Seva \& Ferrando, 2006), partiendo del método de selección de factores. Se empleó el SPSS 22.0 para analizar las propiedades métricas de cada ítem, mediante los coeficientes descriptivos básicos (media, dispersión, curtosis y asimetría). En segundo lugar, se exploró y utilizó el FACTOR, en el cual el diagnóstico de la bondad de ajuste es esencial para establecer la validez de la escala. La evaluación se basó en varios criterios, como recomiendan Bentler (1990), McDonald y Marsh (1990) y Freiberg, Ledesma y Fernández (2017). Con la finalidad de verificar y determinar la consistencia interna del instrumento y de las distintas dimensiones, se usó el coeficiente alpha de Cronbach. También se empleó IBM AMOS® 23 para confirmar la adecuación del instrumento a las poblaciones investigadas, partiendo del método de estimación de parámetros y realizando un análisis multigrupo, con el fin de definir las relaciones entre el autoconcepto global y sus dimensiones, en función de la tendencia religiosa de la muestra. Para este propósito, se empleó el siguiente modelo de ecuaciones estructurales (Figura 1).

\section{Figura 1}

Modelo teórico de autoconcepto

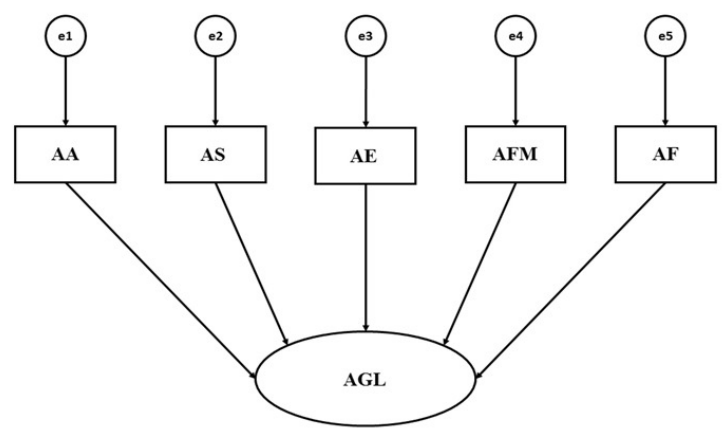

Nota. AGL: Autoconcepto Global; AA: Autoconcepto Académico; AS: Autoconcepto Social; AE: Autoconcepto emocional; AFM: Autoconcepto Familiar; AF: Autoconcepto Físico.

Las variables observables y endógenas del modelo de rutas son: el autoconcepto académico (AA), autoconcepto físico (AF), autoconcepto social (AS), autoconcepto emocional (AE), autoconcepto familiar (AFA). La variable latente y exógena queda representada por el autoconcepto global (AGL). Para estimar las relaciones entre variables, se empleó el método de máxima verosimilitud por ser consistente, no sesgado e invariante en relación con el tipo de escala, considerando que todas las variables tienen una distribución normal.

El ajuste del modelo se comprobó con el fin de verificar la compatibilidad del mismo y la información empírica obtenida. La fiabilidad del ajuste se efectuó con base en los criterios de bondad de ajuste (Marsh, 2007). En el caso del chi-cuadrado, los valores no significativos asociados a p indican un buen ajuste del modelo. El valor del índice de ajuste comparativo (CFI) será aceptable con valores superiores a 0.9 y excelente para valores superiores a 0.95. El índice de ajuste normalizado (NFI) deberá ser superior a 0.9. El valor del índice de incremento de ajuste (IFI) será aceptable con valores superiores a 0.9 y excelente para valores superiores a 0.95. Por último, el valor del error cuadrático medio de aproximación (RMSEA) será excelente si es inferior a 0.05 y aceptable si es inferior a 0.08.

\section{Resultados}

En esta primera parte del análisis, se procede a describir a los participantes según su tendencia religiosa y sexo. Se aprecia cómo en los campus de Granada y Ceuta, las muestras son similares por género, sin embargo, en la ciudad autónoma de Melilla, se observan diferencias estadísticamente significativas, donde un $23 \%$ de las mujeres se consideran musulmanas frente al $10 \%$ de hombres, situación que se invierte en los ateosagnósticos (30 \% frente al $16.8 \%$ ), tal y como se percibe en la Tabla 1. 
Tabla 1

Distribución de la muestra y datos sociodemográficos

\begin{tabular}{llrrrrr}
\hline \multirow{2}{*}{$\begin{array}{c}\text { Campus } \\
\text { Universitario }\end{array}$} & \multirow{2}{*}{ Género } & \multicolumn{5}{c}{ Tendencia Religiosa } \\
\cline { 3 - 6 } & & $\begin{array}{c}\text { Cristiana } \\
n(\%)\end{array}$ & $\begin{array}{c}\text { Musulmana } \\
n(\%)\end{array}$ & $\begin{array}{c}\text { Ateo-Agnóstica } \\
n(\%)\end{array}$ & $\begin{array}{c}\text { Otras } \\
n(\%)\end{array}$ & Total \\
\hline Campus & Hombre & $9(47)$ & $2(11)$ & $8(42)$ & $0(0)$ & 19 \\
Granada & Mujer & $42(50)$ & $10(12)$ & $31(37)$ & $1(1)$ & 84 \\
\hline Campus & Hombre & $17(46)$ & $1(3)$ & $18(48)$ & $1(3)$ & 37 \\
Ceuta & Mujer & $51(50)$ & $15(15)$ & $34(34)$ & $1(1)$ & 101 \\
\hline Campus & Hombre & $57(57)$ & $10(10)$ & $30(30)$ & $3(3)$ & 100 \\
Melilla & Mujer & $152(59)$ & $59(23)$ & $43(17)$ & $2(1)$ & 256 \\
\hline
\end{tabular}

A continuación, para determinar el Análisis Factorial Exploratorio (AFE) se muestran los valores descriptivos del cuestionario AF-5 (Tabla 2). Siguiendo los pasos recomendados por los expertos (Schmider, Ziegler, Danay, Beyer, \& Bühner, 2010), se toma la decisión de prescindir de dos variables (V 19 y V 29), ya que presentan cifras superiores a 2 en las pruebas de dispersión (asimetría y curtosis).

\section{Tabla 2}

Descriptivos de los ítems del AF-5

\begin{tabular}{|c|c|c|c|c|c|c|}
\hline & Media & $D E$ & Varianza & Asimetria & Curtosis & Rango \\
\hline V01 & 4 & 0.6 & 0.447 & -0.4 & 0.619 & 4 \\
\hline$V 02$ & 3.8 & 0.9 & 0.858 & -0.536 & -0.19 & 4 \\
\hline$V 03$ & 3.3 & 0.9 & 0.869 & 0.015 & -0.146 & 4 \\
\hline V 04 & 3.8 & 1.1 & 1.284 & -0.88 & 0.142 & 4 \\
\hline$V 05$ & 3.4 & 1 & 1.009 & -0.16 & -0.482 & 4 \\
\hline$V 06$ & 3.7 & 0.8 & 0.651 & -0.189 & -0.238 & 4 \\
\hline V07 & 4.2 & 0.7 & 0.621 & -0.875 & 0.321 & 4 \\
\hline$V 08$ & 3.3 & 1 & 1.172 & -0.071 & -0.882 & 4 \\
\hline$V 09$ & 4.1 & 0.8 & 0.791 & -1.037 & 0.731 & 3 \\
\hline$V 10$ & 2.9 & 1.3 & 1.742 & 0.031 & -1.126 & 4 \\
\hline V 11 & 3 & 1 & 1.084 & -0.213 & -0.402 & 4 \\
\hline V 12 & 3.9 & 1 & 1.14 & -0.772 & -0.133 & 4 \\
\hline V 13 & 3.4 & 1 & 1.052 & -0.42 & -0.253 & 4 \\
\hline V 14 & 4.1 & 1.5 & 2.277 & -1.439 & 0.231 & 4 \\
\hline$V 15$ & 3.5 & 0.9 & 0.999 & -0.345 & -0.274 & 4 \\
\hline$V 16$ & 3.4 & 0.8 & 0.728 & -0.244 & 0.082 & 4 \\
\hline V 17 & 4.2 & 0.7 & 0.551 & -0.699 & 0.077 & 4 \\
\hline$V 18$ & 3.4 & 1 & 1.083 & -0.388 & -0.423 & 4 \\
\hline$V 19$ & 4.6 & 0.7 & 0.555 & -2.223 & 4.675 & 3 \\
\hline$V 20$ & 3.4 & 1.1 & 1.24 & -0.41 & -0.465 & 4 \\
\hline$V 21$ & 3.8 & 0.7 & 0.629 & -0.312 & -0.141 & 4 \\
\hline$V 22$ & 3.5 & 1.1 & 1.254 & -0.326 & -0.636 & 4 \\
\hline V 23 & 2.8 & 1.2 & 1.449 & 0.026 & -0.892 & 4 \\
\hline V 24 & 4.3 & 0.9 & 0.859 & -1.25 & 0.858 & 4 \\
\hline$V 25$ & 3.2 & 1.2 & 1.476 & -0.198 & -0.849 & 4 \\
\hline$V 26$ & 3.5 & 0.8 & 0.713 & -0.25 & -0.037 & 4 \\
\hline$V 27$ & 3.6 & 0.9 & 0.968 & -0.347 & -0.446 & 4 \\
\hline V 28 & 2.7 & 1 & 1.082 & 0.421 & -0.161 & 4 \\
\hline$V 29$ & 4.6 & 0.7 & 0.558 & -2.166 & 4.459 & 4 \\
\hline V 30 & 3.4 & 0.9 & 0.992 & -0.352 & -0.072 & 4 \\
\hline
\end{tabular}

Seguidamente, mediante la utilización del programa FACTOR Analysis (Lorenzo-Seva \& Ferrando, 2006), se rotaron cinco factores. El estadístico de Bartlett [6477.9 ( $g l=435 ; p<$
0.001)] y el test de Kaiser-Meyer-Olkin (KMO) $=0.822$, utilizados para probar si la muestra procede de poblaciones con la misma varianza y si presenta una buena adecuación muestral, indican un buen ajuste de los datos para ser sometidos al análisis factorial. Los cinco factores extraídos explican el $52 \%$ de la varianza total, el índice de bondad de ajuste (GFI) fue de 0.98, el índice AGFI fue de 0.97 y la raíz cuadrática media de los residuales (RMSR) de 0.04. Todos estos datos indican un buen ajuste para estos ítems. Como se determina en la Tabla 3, desaparecieron dos variables (V 20 y $\mathrm{V}$ 30), por tener cargas inferiores a $0.3, y$ la V 15 por cargar en un factor que no le correspondía. La escala definitiva quedó formada por cinco factores. El primero conformado por seis variables se corresponde con la dimensión emocional; el segundo factor constituido por seis variables se relaciona con la dimensión social; el tercero, donde se agrupan cuatro variables, coincide con la dimensión familiar; un cuarto que se corresponde con la dimensión académica, formado por seis variables y el quinto y último factor constituido por tres variables, se concierne a la dimensión física. También se muestra la configuración del cuestionario con un buen ajuste, el coeficiente de fiabilidad fue de 0.794, y suprimidas las variables, por factores se obtuvo un 0.746 para el Factor 1 (emocional), 0.787 para el Factor 2 (social), 0.705 para el Factor 3 (familiar), 0.808 para el Factor 4 (académico) y 0.787 en el Factor 5 (físico). 
Tabla 3

Matriz Factorial Rotada y carga factorial del cuestionario

\begin{tabular}{|c|c|c|c|c|c|c|c|c|c|c|c|}
\hline & \multicolumn{5}{|c|}{$\begin{array}{l}\text { Matriz Factorial Rotada (omitiendo las cargas } \\
\text { inferiores a 0.3) }\end{array}$} & \multicolumn{6}{|c|}{ Carga factorial de las dimensiones del AF5 } \\
\hline & & & F3 & $\mathrm{F} 4$ & & & F1 & $\mathrm{F} 2$ & F3 & F4 & F5 \\
\hline V0I & -0.018 & -0.011 & 0.03 & 0.564 & -0.071 & V03 & 0.528 & & & & \\
\hline $\mathrm{V} 02$ & -0.093 & 0.912 & -0.06 & -0.038 & -0.051 & $V O S$ & 0.605 & & & & \\
\hline V03 & 0.528 & -0.086 & 0.035 & -0.046 & 0.07 & $V 13$ & 0.531 & & & & \\
\hline V04 & 0.126 & -0.045 & 0.521 & -0.049 & -0.019 & $V 18$ & 0.614 & & & & \\
\hline vos & -0.028 & -0.038 & 0.016 & 0.155 & 0.568 & $V 23$ & 0.531 & & & & \\
\hline V06 & -0.044 & -0.067 & -0.006 & 0.867 & -0.048 & $V 28$ & 0.625 & & & & \\
\hline V07 & -0.158 & 0.596 & 0.011 & 0.175 & 0.056 & $\mathrm{~V} 02$ & & 0.912 & & & \\
\hline Vos & 0.605 & -0.108 & -0.002 & 0.031 & 0.045 & $V 07$ & & 0.596 & & & \\
\hline V09 & .0002 & 0.109 & 0.656 & 0.029 & 0.038 & $V 12$ & & 0.771 & & & \\
\hline V10 & -0.035 & 0.026 & -0.025 & -0.09 & 0.752 & $V 17$ & & 0.442 & & & \\
\hline VII & -0.123 & 0.109 & -0.072 & 0.366 & 0.031 & $V 22$ & & 0.468 & & & \\
\hline$V 12$ & 0.093 & 0.771 & 0.083 & -0.138 & -0.124 & $V 27$ & & 0.558 & & & \\
\hline$V 13$ & 0.531 & -0.008 & -0.001 & -0.092 & 0.166 & V04 & & & 0.521 & & \\
\hline V14 & 0.093 & 0.003 & & & & $V 09$ & & & 0.656 & & \\
\hline VIS & -0.035 & 0.166 & -0.023 & & & $V 14$ & & & & & \\
\hline V16 & 0.063 & 0.113 & -0.052 & 0.617 & & V 24 & & & 0.786 & & \\
\hline V17 & -0.023 & 0.44 & 0.0 & 0.17 & & $\mathrm{VOI}$ & & & & 0.564 & \\
\hline V18 & 0.614 & 0.111 & 0.02 & 0.006 & -0.087 & $V 06$ & & & & 0.867 & \\
\hline$V 20$ & 0.18 & & & 0.19 & & $V 11$ & & & & 0.3 & \\
\hline$V 21$ & 0.049 & -0.125 & 0.071 & & -0.0 & $V 16$ & & & & 0.617 & \\
\hline$V 22$ & 0.316 & 0.468 & -0.044 & -0.092 & -0.083 & $V 21$ & & & & 0.75 & \\
\hline$V 23$ & 0.531 & & -0.167 & 0.111 & -0.0 & $V 26$ & & & & 0.809 & \\
\hline$V 24$ & -0.09 & & & & & V05 & & & & & 0.568 \\
\hline$V 25$ & 0.021 & & -0.0 & -0.1 & & $V 10$ & & & & & 0.752 \\
\hline$V 26$ & 0.028 & -0.093 & 0.019 & 0.809 & -0.003 & $V 25$ & & & & & 0.944 \\
\hline$V 27$ & -0.092 & 0.558 & 0.053 & -0.045 & 0.168 & & & & & & \\
\hline$V 28$ & 0.625 & 0.042 & 0.073 & -0.001 & -0.028 & & & & & & \\
\hline$V 30$ & 0.147 & 0.125 & 0.021 & 0.221 & 0.292 & & & & & & \\
\hline$\alpha$ & & & & & & 0.794 & 0.746 & 0.787 & 0.705 & 0.808 & 0.787 \\
\hline
\end{tabular}

Como se observa en la tabla, el alpha de Cronbach resultó satisfactorio en su totalidad y en cada uno de los factores presentó valores $\alpha>$ 0.7 (Tabla 3). En relación con las correlaciones bivariadas de Pearson entre las dimensiones, como se muestra en la Tabla 4, se aprecia que casi todos los factores presentan correlación significativa en un $99 \%,(p<0.01)$, siendo los valores más elevados los que relacionan entre sí la dimensión social, familiar y académica $(r \geq$ $0.2 * *)$.

\section{Tabla 4}

Correlación de las dimensiones del AF-5

\begin{tabular}{|c|c|c|c|c|c|}
\hline & Factor 1 & Factor 2 & Factor 3 & Factor 4 & Factor 5 \\
\hline Factor 1: Dimensión Emocional & 1 & & & & \\
\hline Factor 2: Dimensión Social & $0.191^{* *}$ & 1 & & & \\
\hline Factor 3: Dimensión Familiar & -0.047 & $0.256^{\circ *}$ & 1 & & \\
\hline Factor 4: Dimensión Académico & $0.08^{*}$ & $0.271^{* *}$ & $0.269^{* *}$ & 1 & \\
\hline Factor 5: Dimensión Física & 0.079 & $0.216^{* *}$ & $0.203^{* *}$ & $0.146^{* *}$ & 1 \\
\hline
\end{tabular}

Habiéndose comprobado la adecuación del AFE, se realizó el Análisis Factorial Confirmatorio (AFC) para ratificar la adecuación del instrumento a la población objeto de estudio, según la tendencia religiosa, empleando el programa AMOS 23.0. Los valores obtenidos en el modelo de ecuaciones estructurales indican un buen ajuste en todos los índices. El chi-cuadrado presentó un valor significativo de $\mathrm{p}\left(\chi^{2}=46.482\right.$; $g l=15 ; p<0.001)$, aunque debe considerarse que este estadístico, como índice, no tiene límite superior. Asimismo, no se puede interpretar de manera estandarizada, además del problema que plantea su sensibilidad al tamaño muestral. De este modo, se emplearon otros índices de ajuste estandarizados que son menos sensibles al tamaño muestral. El índice de ajuste comparativo (CFI) mostró un valor de 0.932, el cual es aceptable. El índice de ajuste normalizado (NFI) reveló un valor de 0.907 y el índice de incremento de ajuste (IFI) un valor de 0.945 , los cuales fueron también apropiados. Finalmente, el error cuadrático medio de aproximación (RMSEA) obtuvo un valor aceptable de 0.06 .

Las Figuras 2, 3 y 4 revelan los valores estimados de los parámetros del modelo en función de la tendencia religiosa. Su magnitud ha de ser adecuada y los efectos significativamente distintos de cero. Tampoco se deben obtener estimaciones impropias como varianzas negativas.

\section{Figura 2}

Autoconcepto según tendencia cristiana

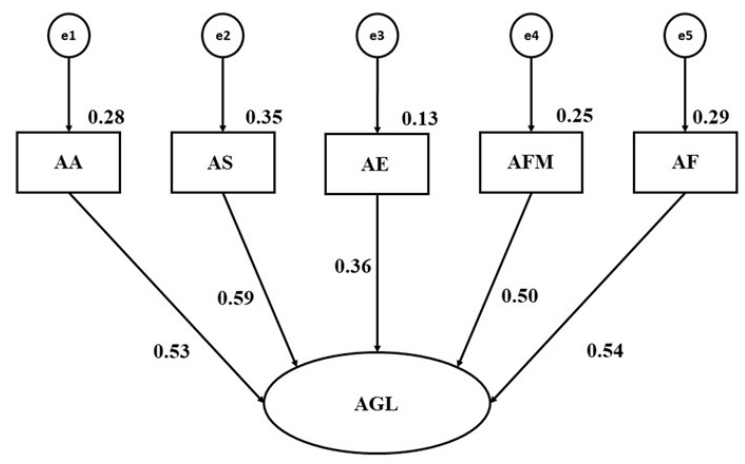

Nota. AGL: Autoconcepto Global; AA: Autoconcepto Académico; AS: Autoconcepto Social; AE: Autoconcepto emocional; AFM: Autoconcepto Familiar; AF: Autoconcepto Físico. 
Figura 3

Autoconcepto según musulmana

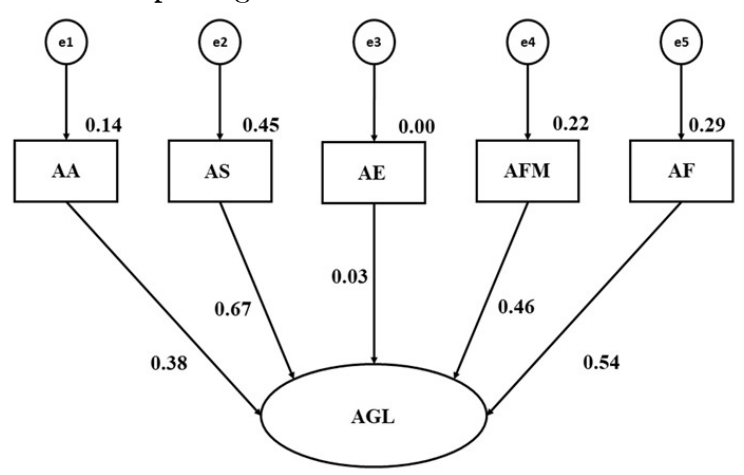

Nota. AGL: Autoconcepto Global; AA:

Autoconcepto Académico; AS: Autoconcepto

Social; AE: Autoconcepto emocional; AFM:

Autoconcepto Familiar; AF: Autoconcepto Físico.

\section{Figura 4}

Autoconcepto según tendencia ateo-agnostica

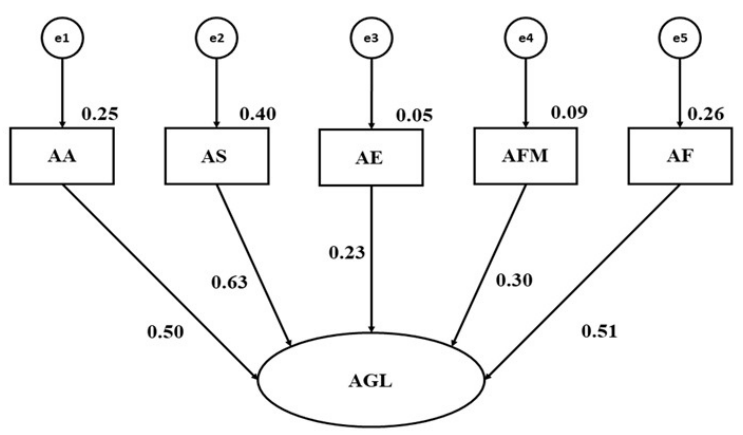

Nota. AGL, Autoconcepto Global; AA, Autoconcepto Académico; AS, Autoconcepto

Social; AE, Autoconcepto emocional; AFM,

Autoconcepto Familiar; AF, Autoconcepto Físico.

A continuación, se muestran los pesos de regresión de las relaciones entre autoconcepto global y sus diferentes dimensiones, en función de la tendencia religiosa (Tabla 5). En el caso de los universitarios con tendencia cristiana, se obtuvieron relaciones estadísticamente significativas al nivel $p<0.001$ para todas las asociaciones, mientras que para las tendencias islámica y atea-agnóstica el nivel de significatividad oscila entre $p<0.05$ y $p<0.001$.

Detallando los pesos de regresión estandarizados del modelo en relación con el autoconcepto global, el mayor peso de regresión para el autoconcepto académico fue el mostrado por la tendencia cristiana $(r=$
0.528), seguido de la atea-agnóstica $(r=$ $0.499)$ y la musulmana $(r=0.376)$. Para la dimensión social, la relación de mayor fortaleza se observa para la tendencia islámica $(r=$ $0.668)$ y la menor para la cristiana $(r=0.528)$. Asimismo, los universitarios cristianos fueron aquellos en quienes el autoconcepto dependía más de la dimensión emocional $(r=0.358)$, seguidos de los ateos-agnósticos $(r=0.23)$. En el caso de los musulmanes, no se hallaron diferencias estadísticamente significativas para esta dimensión. El autoconcepto familiar reveló el mayor peso de regresión para los cristianos $(r=$ 0.502), seguidos de los musulmanes $(r=0.464)$ y de los ateos-agnósticos con la menor relación $(r$ $=0.3)$. Finalmente, el autoconcepto físico revela el mismo valor para cristianos y musulmanes $(r=$ 0.543), mientras que los ateos-agnósticos reflejan un valor inferior $(r=0.512)$.

\section{Tabla 5}

Pesos de regresión y pesos de regresión estandarizados

\begin{tabular}{|c|c|c|c|c|c|c|}
\hline \multicolumn{3}{|c|}{ Relaciones entre variables } & P. R. Estimaciones & S.E. & R. C. & P. R. E. Estimaciones \\
\hline \multicolumn{7}{|c|}{ Tendencia cristiana } \\
\hline AA & $\leftarrow$ & AGL & 1 & - & - & $0.528^{* * *}$ \\
\hline AS & $\leftarrow$ & AGL & 1.193 & 0.202 & 5.917 & $0.591^{* * *}$ \\
\hline $\mathrm{AE}$ & $\leftarrow$ & AGL & 0.511 & 0.114 & 4.481 & $0.358^{* * * *}$ \\
\hline AFM & $\leftarrow$ & AGL & 0.932 & 0.168 & 5.556 & $0.502^{* * *}$ \\
\hline $\mathrm{AF}$ & $\leftarrow$ & AGL & 1.256 & 0.218 & 5.757 & $0.543^{* * *}$ \\
\hline \multicolumn{7}{|c|}{ Tendencia islámica } \\
\hline AA & $\leftarrow$ & AGL & 1 & - & - & $0.376^{*}$ \\
\hline AS & $\leftarrow$ & AGL & 2.006 & 0.82 & 2.447 & $0.668^{*}$ \\
\hline $\mathrm{AE}$ & $\leftarrow$ & AGL & 0.051 & 0.228 & 0.224 & 0.029 \\
\hline AFM & $\leftarrow$ & AGL & 1.33 & 0.568 & 2.343 & $0.464^{*}$ \\
\hline $\mathrm{AF}$ & $\leftarrow$ & AGL & 1.895 & 0.77 & 2.46 & $0.543^{*}$ \\
\hline \multicolumn{7}{|c|}{ Tendencia atea o agnóstica } \\
\hline AA & $\leftarrow$ & AGL & 1 & - & - & $0.499^{* * *}$ \\
\hline AS & $\leftarrow$ & AGL & 1.445 & 0.41 & 3.529 & $0.633^{* * *}$ \\
\hline $\mathrm{AE}$ & $\leftarrow$ & AGL & 0.35 & 0.167 & 2.094 & $0.23^{*}$ \\
\hline AFM & $\leftarrow$ & AGL & 0.683 & 0.264 & 2.585 & $0.3^{* *}$ \\
\hline $\mathrm{AF}$ & $\leftarrow$ & AGL & 1.265 & 0.356 & 3.552 & $0.512^{* * *}$ \\
\hline
\end{tabular}

Nota. P. R.: Pesos de Regresión; P. R. E.: Pesos de Regresión Estandarizados; E. E.: Errores de Estimación; R. C.: Ratio Crítico. AGL: Autoconcepto Global; AA: Autoconcepto Académico; AS: Autoconcepto Social; AE: Autoconcepto emocional; AFM: Autoconcepto Familiar; AF: Autoconcepto Físico. $* p<0.05 ; * * p<0.01 ; * * *<0.001$.

\section{Discusión}

Para establecer la evaluación psicosocial y religiosa en el contexto universitario, se considera imprescindible disponer de cuestionarios que reporten datos fiables y reales 
de lo que se quiere medir. Por tanto, el principal objetivo de este trabajo fue analizar las propiedades psicométricas del cuestionario AF-5 y observar su adaptación y aplicación a la población universitaria con diversas tendencias religiosas.

La población objeto de estudio reúne unas características que lo diferencian del resto (Gálvez-Nieto, Polanco, \& Salvo, 2016; Medina, 2006; Rodríguez, 2008), ya que las ciudades de Ceuta y Melilla son poblaciones transfronterizas y multiculturales donde residen y conviven ciudadanos de las principales tendencias religiosas actualmente existentes. A este respecto, tanto en las ciudades de Granada como Ceuta, se obtienen cifras parecidas por género y tendencia religiosa, mientras que en Melilla, las mujeres se consideran musulmanas en mayor proporción que los hombres, invirtiéndose las cifras en el caso de los ateos-agnósticos. Estos valores indican que los hombres tienen una menor tendencia a las creencias religiosas, argumentado por los estereotipos de género donde, según Castillo-Mayén y Montes-Berges (2014), aspectos como la religiosidad vienen representados por diferencias entre hombres y mujeres.

Los resultados obtenidos fueron satisfactorios en cuanto a los coeficientes alpha de Cronbach, tanto en la totalidad de la escala como para los cinco factores, mostrándose el AF-5 como un instrumento válido y fiable para medir la autopercepción (autoconcepto) de forma multidimensional, al igual que señalaron otros autores (Molero, Ortega-Álvarez, Valiente, \& Zagalaz-Sánchez, 2010).

Los resultados se mantienen estables en las dimensiones académica, social y emocional, cargando los factores de una forma coherente, mientras que en las dimensiones familiar y física, se pierde alguna variable. Entendemos que la población estudiada (mayoría de mujeres) siente una menor predisposición hacia la dimensión física (Castro, Zurita, Martínez, Chacón, \& Espejo, 2016), si bien este test ha sido ampliamente aplicado en el contexto deportivo (Frutos-de-Miguel, 2018; Murgui, García, \& García, 2016; Zurita et al., 2016) y familiar, encontrándose en algunos casos fuera del domicilio familiar (Álvarez et al., 2015).

Como ya se ha comentado, los valores hallados confirman la teoría de la multidimensionalidad propuesta por Shavelson et al. (1976) y replican el modelo, presentando unos índices de ajuste aceptables y con una buena consistencia interna y estabilidad temporal. Asimismo, se presentan valores que superan el 0.7 y se ajustan de manera correcta en todas las dimensiones, lo que muestra una fiabilidad similar a otros estudios (Murgui, García, García, \& García, 2012) y genera una mayor perspectiva que la mostrada por los instrumentos que solamente miden la dimensión física (Marsh, Bar-Eli, Zach, \& Richards, 2006) o académica (RamosDíaz, Rodríguez-Fernández, Fernández-Zabala, Revuelta, \& Zuazagoitia, 2016). No obstante, las cifras arrojadas sugieren algunas cuestiones que deberían ser atendidas en futuros trabajos.

En el presente trabajo se encontró una alta correlación entre la dimensión social, familiar y académica, entendiendo que esta relación puede estar generada por el contexto y perfil sociológico donde se realizó la investigación, que se caracteriza por personas que realizan estudios superiores universitarios, en algunos casos residentes fuera del domicilio familiar y con una vitalidad social propia de personas jóvenes (Chacón-Cuberos et al., 2017).

$\mathrm{Si}$ se considera la tendencia religiosa, los resultados revelan que la influencia que ejerce el autoconcepto académico en el autoconcepto global es más elevada en los cristianos, influyendo en menor medida en el colectivo musulmán. Sin embargo, se presenta la situación inversa para la dimensión social, la cual muestra un peso de regresión mayor en los musulmanes. Estas premisas son fundamentadas por Pulido y Herrera (2015) y Siqués y Vila (2014), quienes adjudican esta diversidad a los núcleos familiares y los diferentes estilos de crianza, ya que cada etnia presenta una mayor implicación hacia una dimensión.

En los estudiantes cristianos, la dimensión emocional influye más en el autoconcepto global que en los ateos y agnósticos, no hallándose influencia para la tendencia musulmana; lo que 
se puede entender si unimos las emociones a las convicciones religiosas que ofrecen a la persona seguridad y sentido último y operan de guía moral y espiritual (Martín-Velasco, 1992), generando un aumento en el ámbito emocional.

La dimensión familiar y física influyen en mayor medida en los estudiantes con alguna tendencia religiosa que en los ateos-agnósticos. Al hilo de este hallazgo, Pérez-Delgado (1992) señala que los ateos-agnósticos prefieren valores asociados a una vida confortable, excitante o agradable, mientras que sujetos con otras tendencias religiosas muestran interés por la autorrealización, la seguridad familiar o la independencia (Exline et al., 2014; Grubbs et al., 2016).

Desde el punto de vista metodológico, este modelo apoya el uso de este cuestionario hacia la actitud religiosa en universitarios, aunque presenta algunas limitaciones. En primer lugar, la muestra del estudio no ha sido homogénea a todas las creencias religiosas, por lo que en futuros trabajos debería explorarse la estructura de otras religiones (hinduismo, budismo, etc.). En segundo lugar, se podría utilizar para establecer las posibles diferencias con otras variables (p. ej., género o nivel educativo).

Como conclusión, los resultados de este estudio apoyan la idea del AF-5 como test válido y fiable para medir de forma satisfactoria el autoconcepto en universitarios de diferente tendencia religiosa, pudiendo analizarlos de manera multidimensional. Asimismo, el estudio del autoconcepto en función de la tendencia religiosa reveló que las dimensiones académica y emocional presentan una mayor fortaleza en los cristianos, mientras que la social lo hace para los musulmanes. Del mismo modo, la dimensión familiar y física es más elevada para los universitarios que profesan alguna religión.

\section{Referencias}

Álvarez, A., Suárez, N., Tuero, E., Núñez, J. C., Valle, A., \& Regueiro, B. (2015). Implicación familiar, autoconcepto del adolescente y rendimiento académico.
European Journal of Investigation in Health, Psychology and Education, 5(3), 293-311. ht tps://doi.org/10.1989/ejihpe.v5i3.133

Bentler, P. M. (1990). Comparative fit indexes in structural models. Psychological Bulletin, 107(2), 238-246. https://doi.org/10.1037/0 033-2909.107.2.238

Castillo-Mayén, R., \& Montes-Berges, B. (2014). Análisis de los estereotipos de género actuales. Anales de Psicología, 30(3), 1044-1060. https://doi.org/10.6018/analesp s.30.3.138981

Castro, M., Zurita, F., Martínez, A., Chacón, R., \& Espejo, T. (2016). Clima motivacional de los adolescentes y su relación con el género, la práctica de actividad física, la modalidad deportiva, la práctica deportiva federada y la actividad física familiar. Revista Internacional de Ciencias del Deporte, 12 (45), 262-277. https://doi.org/10.5232/ricyde201 6.04504

Chacón-Cuberos, R., Zurita, F., Castro, M., Espejo, T., Martínez, A., \& Lucena, M. (2017). Análisis descriptivo del consumo de sustancias nocivas, adhesión a la dieta mediterránea y tipo de residencia en estudiantes universitarios de Granada. Revista Complutense de Educación, 28(3), 33-47. Recuperado de http://revistas.ucm.e s/index.php/RCED/article/view/50083

Elzo, J., \& González-Anleo, J. (1999). Los jóvenes y la religión. En J. Elzo (Coord.), Jóvenes españoles 99 (pp. 263-354). Madrid: Fundación Santa María.

Exline, J. J., Pargament, K. I., Grubbs, J. B., \& Yali, A. M. (2014). The religious and spiritual struggles scale: Development and initial validation. Psychology of Religion and Spirituality, 6(3), 208-222. https://doi.org/1 $0.1037 / \mathrm{a} 0036465$

Fernández-Zabala, A., Rodríguez-Fernández, A., \& Goñi, A. (2016). The structure of the Social Self Concept (SSC) questionnaire. Anales de Psicología, 32 (1), 199-205. https:/ /doi.org/10.6018/analesps.32.1.193931

Flores, P. E., Medrano, L. A., \& Manoiloff, L. M. (2014). Estado de ánimo y juicios de autoconcepto en universitarios: análisis 
Félix Zurita-Ortega, Silvia San Román-Mata, Asunción Martínez-Martínez, et al.

desde un abordaje basado en redes semánticas naturales. Revista Interamericana de Psicología, 48(2), 291-307. Recuperado de https://journal.sipsych.org/index.php/IJ $\mathrm{P} /$ article/viewFile/268/pdf_41

Frutos-de-Miguel, J. (2018). Physical selfconcept as a tool for social inclusion in the physical education area. Journal of Sport and Health Research, 10(1), 25-42. Recuperado de http://www.journalshr.com/papers/ $\mathrm{Vol}$ \%2010_N\%201/JSHR\%20V10_1_3.pdf

Freiberg, A., Ledesma, R., \& Fernández, M. M. (2017). Análisis de las propiedades psicométricas del Inventario de Estrategias de Aprendizaje y Estudio (LASSI) en estudiantes universitarios. Revista Iberoamericana de Diagnóstico y Evaluación Psicológica, 44(2), 116-130. https://doi.org/ 10.21865/RIDEP44.2.10

Gálvez-Nieto, J. L., Polanco, K., \& Salvo, S. (2016). Propiedades psicométricas de la Escala de Autoconcepto Académico (EAA) en estudiantes chilenos. Revista Iberoamericana de Diagnóstico y Evaluación Psicológica, 43(1), 5-16. https://doi.org/10.2 1865/RIDEP43 5

García, F., \& Musitu, G. (1999). AF5. Autoconcepto Forma 5. Madrid: TEA.

García, F., Gracia, E., \& Zeleznova, A. (2013). Validation of the English version of the Five-Factor Self-Concept Questionnaire. Psicothema, 25 (4), 549-555. https://doi.org/ 10.7334/psicothema2013.33

González, M., \& Ibáñez, I. (2018). Propiedades psicométricas de una versión española breve de 30 ítems del Cuestionario de Ansiedad y Depresión (MASQE30). Universitas Psychologica, 17(1), 1-10. https:/ /doi.org/10.11144/Javeriana.upsy17-1.ppve

Grubbs, J. B., Wilt, J., Stauner, N., Exline, J. J., \& Pargament, K. I. (2016). Self-struggle, and soul: Linking personality, self-concept, and religious/spiritual struggle. Personality and Individual Differences, 101, 144-152. ht tps://doi.org/10.1016/j.paid.2016.05.365

Lobos, K., Díaz, A., Bustos, C., \& Pérez, M. V. (2015). Construction and psychometric characteristic of the Self-Concept Scale of interaction in the classroom. Psicothema, 27(2), 151-158. https://doi.org/10.7334/psi cothema2014.224

Lohbeck, A., Nitkowski, D., \& Petermann, F. (2016). A control value theory approach: Relationships between academic selfconcept, interest, and test anxiety in elementary school children. Child $\mathbb{E}$ Youth Care Forum, 45(6), 887-904. https://doi.org /10.1007/s10566-016-9362-1

López, A. B., Huynh, V. W., \& Fuligni, A. J. (2011). A longitudinal study of religious identity and participation during adolescence. Child Development, 82(4), 1297-1309. https://doi.org/10.1111/j.14678624.2011.01609.x

Lorenzo-Seva, U., \& Ferrando, P. J. (2006). FACTOR: A computer program to fit the exploratory factor analysis model. Behavioral Research Methods, Instruments and Computers, 38(1), 88-91. https://doi.or $\mathrm{g} / 10.3758 / \mathrm{BF} 03192753$

Marsh, H. W. (1990). The structure of academic self-concept: The Marsh/Shavelson model. Journal of Educational Psychology, 82(4), 623-636. https://doi.org/10.1037/0022-066 3.82.4.623

Marsh, H. W. (2007). Handbook of sport psychology (3.a ed.). Hoboken, NJ: G. Tenenbaum and R. C. Eklund.

Marsh, H., Bar-Eli, M., Zach, S., \& Richards, G. (2006). Construct validation of Hebrew versions of three physical selfconcept measures and extended multitraitmultimethod analysis. Journal of Sport and Exercise Psychology, 28(3), 310-343. https:// doi.org/10.1123/jsep.28.3.310

Martín-Velasco, J. (1992). Religión y moral. En M. Vidal (Ed.), Conceptos fundamentales de ética teológica (pp. 185-203). Madrid: Trotta.

McDonald, R. P., \& Marsh, H. W. (1990). Choosing a multivariate model: Noncentrality and goodness of fit. Psychological Bulletin, 107(2), 247-255. http s://doi.org/101037/0033-2909.107.2.247

Medina, M. L. (2006). Convivencia en Melilla: sus cuatro culturas. En E. Soriano, A. 
J. González \& M. M. Osorio (Coords.), Convivencia y mediación intercultural (pp. 79-85). Almería, ES: Universidad de Almería.

Molero, D., Ortega-Álvarez, F., Valiente, I., \& Zagalaz-Sánchez, M. L. (2010). Estudio comparativo del autoconcepto físico en adolescentes en función del género y del nivel de actividad físico-deportiva. Retos. Nuevas tendencias en Educación Física, Deporte y Recreación, 17, 38-41. Recuperado de http://www.redalyc.org/pdf/3457/34573 2283008.pdf

Murgui, S., García, C., \& García, A. (2016). Efectos de la práctica deportiva en la relación entre las habilidades motoras, el autoconcepto físico y el autoconcepto multidimensional. Revista de Psicología del Deporte, 25(1), 19-25. Recuperado de http://www.rpd-online.com/article/view /v25-n1-murgui-garcia-garcia

Murgui, S., García, C., García, A., \& García, F. (2012). Autoconcepto en jóvenes practicantes de danza y no practicantes: Análisis factorial confirmatorio de la escala AF5. Revista de Psicología del Deporte, 21 (2), 263-269. Recuperado de http://www.rpd-o nline.com/article/view/727

Núñez, J. L., Martín-Albo, J., Navarro, J. G., \& Grijalvo, F. (2007). Análisis de las propiedades psicométricas del cuestionario Autoconcepto Forma 5 en estudiantes universitarios. Estudios de Psicología, 28(3), 333-342. https://doi.org/10.1174/02109390 7782506461.

Pargament, K. I. (2002). The bitter and the sweet: An evaluation of the costs and benefits of religiousness. Psychological Inquiry, 13(3), 168-181. https://doi.org/10.1 207/S15327965PLI1303_02

Pérez-Delgado, E. (1992). Religión y moral en jóvenes adolescentes y adultos españoles. Relaciones empíricas entre religiosidad y moralidad. Teología Espiritual, 36(3), 1-61.

Pérez-Delgado, E. (2000). Moral de convicciones, moral de principios. Una introducción a la ética desde las ciencias humanas. Salamanca: Editorial San Esteban.
Puertas-Molero, P., González-Valero, G., \& Sánchez-Zafra, M. (2017). Influence of sports physical practice on the emotional intelligence of students: A systematic review. Education, Sport, Health and Physical Activity, 1(1), 10-24. Recuperado de http:// hdl.handle.net/10481/48957

Pulido, F., \& Herrera, F. (2015). La inteligencia emocional como predictora del rendimiento académico: el contexto pluricultural de Ceuta. Innoeduca. International Journal of Technology and Education al Innovation, 1(2), 98-105. https://doi.org/10.20548/innoeduc a.2015.v1i2.1033

Ramos-Díaz, E., Rodríguez-Fernández, A., Fernández-Zabala, A., Revuelta, L., \& Zuazagoitia, A. (2016). Apoyo social percibido, autoconcepto e implicación escolar de estudiantes adolescentes. Revista Psicodidáctica, 21 (2), 339-356. https://doi.o $\mathrm{rg} / 10.1387 /$ RevPsicodidact.14848

Rodríguez, G. J. (2008). Las minorías religiosas en Melilla. Revista General de Derecho Canónico y Derecho Eclesiástico del Estado, 17, 5-10. Recuperado de https://dialnet.unirioja.es/se rvlet/articulo? codigo $=2654938$

Salum-Fares, A., Marín, A. R., \& Reyes, A. C. (2011). Relevancia de las dimensiones del Autoconcepto en estudiantes de Escuelas secundarias de Ciudad Victoria, Tamaulipas, México. Revista Electrónica de Psicología Iztacala, 14(2), 255-272. Recuperado de http://www.medigraphic.co m/pdfs/epsicologia/epi-2011/epi112n.pdf

Schmider, E., Ziegler, M., Danay, E., Beyer, L., \& Bühner, M. (2010). Is it really robust? Reinvestigating the robustness of ANOVA against violations of the normal distribution assumption. Methodology, 6, 147-151. https ://doi.org/10.1027/1614-2241/a000016

Shavelson, J., Hubner, J. J., \& Stanton, G. C. (1976). Self-concept: Validation of construct interpretations. Review of Educational Research, 46, 407-441. https://d oi.org/10.3102/00346543046003407

Silvia, L. (2016). Validez, confiabilidad y normas del cuestionario de autoconcepto físico en 
universitarios de Trujillo Metropolitano. Revista de Psicología, 18(1), 9-25. https://do i.org/10.18050/revpsi.v18n1a1.2016

Siqués, C., \& Vila, I. (2014). Estrategias discursivas y aprendizaje de la lengua escolar: un estudio etnográfico en un aula de alta diversidad étnica y lingüística. Cultura y Educación, 26(2), 349- 376. https ://doi.org/10.1080/11356405.2014.935112

Standage, M., \& Treasure, D. C. (2002). Relationship among achieve-ment goal orientations and multidimensional situational motivation in physical education. British Journal of Educational Psychology, 72(1), 87-103. https://doi.org/1 0.1348/000709902158784

Standage, M., Duda, J. L., \& Ntoumanis, N. (2005). A test of self-determination theory in school physical education. British Journal of Educational Psychology, 75, 411-433. http s://doi.org/10.1348/000709904X22359

Tornare, E., Czajkowski, N. O., \& Pons, F. (2015). Children's emotions in math problem solving situations: Contributions of selfconcept, metacognitive experiences, and performance. Learning and Instruction, 39, 88-96. https://doi.org/10.1016/j.learninstru c. 2015.05.011

Wilkins, S., Butt, M. M., Kratochvil, D., \& Balakrishnan, M. S. (2016). The effects of social identification and organizational identification on student commitment, achievement and satisfaction in higher education. Studies in Higher Education, 41 (12), 2232-2252. https://doi.org/10.1080 /03075079.2015.1034258

Worthen, M. G., Lingiardi, V., \& Caristo, C. (2017). The roles of politics, feminism, and religion in attitudes toward LGBT individuals: A cross-cultural study of college students in the USA, Italy, and Spain. Sexuality Research and Social Policy, 14(3), 241-258. https://doi.org/10.1007/s13 178-016-0244-y

Wylie, R. C. (1979). The self-concept, Volume 2: Theory and research on selected topics. Lincoln, NE: University of Nebraska.
Zurita, F., Castro-Sánchez, M., Álvaro, J. I., Rodríguez, S., \& Pérez, A. (2016). Autoconcepto, actividad física y familia: un modelo de ecuaciones estructurales. Revista de Psicología del Deporte, 25(1), 97-104. Recuperado de http://www.rpd-online.com/article/view /v25-n1-zurita-ortega-castro-etal

Zurita, F., Moreno, G., González, G., Viciana, V., Martínez, A., \& Muros, J. J. (2018). Revisión conceptual entre inteligencia emocional y autoconcepto físico. Sport TK. Revista EuroAmericana de Ciencias del Deporte, 7(1), 139-144. https://doi.org/10.6 $018 / 322001$

\section{Notas}

* Artículo de investigación. El presente trabajo no obtuvo financiación externa. 